This document is the Accepted Manuscript version of a Published Work that appeared in final form in Advanced Functional Materials, copyright @ Wiley after peer review and technical editing by the publisher. To access the final edited and published work see DOI: 10.1002/adfm.201906803.

\title{
The Dynamic Emission Zone in Sandwich Polymer Light- Emitting Electrochemical Cells
}

Matthias Diethelm+, Andreas Schiller+, Maciej Kawecki, Andrius Devižis, Balthasar Blülle, Sandra Jenatsch, Evelyne Knapp, Quirin Grossmann, Beat Ruhstaller, Frank Nüesch, and Roland Hany*

+ Authors contributed equally

M. Diethelm, Q. Grossmann, Prof. F. Nüesch, Dr. R. Hany

Empa, Swiss Federal Laboratories for Materials Science and Technology, Laboratory for Functional Polymers, 8600 Dübendorf, Switzerland

E-mail: roland.hany@empa.ch

M. Diethelm, Prof. F. Nüesch

EPFL, Institute of Materials Science and Engineering, Ecole Polytechnique Fédérale de Lausanne, Station 12, 1015 Lausanne, Switzerland

A. Schiller, Dr. E. Knapp, Prof. B. Ruhstaller ZHAW, Zurich University of Applied Sciences, Institute of Computational Physics, Technikumstrasse 9, 8401 Winterthur, Switzerland

A. Schiller, Dr. B. Blülle, Dr. S. Jenatsch, Prof. B. Ruhstaller

Fluxim AG, Katharina-Sulzer-Platz 2, 8400 Winterthur, Switzerland

M. Kawecki

a) Empa, Swiss Federal Laboratories for Materials Science and Technology, Nanoscale Materials Science, 8600 Dübendorf, Switzerland

b) University of Basel, Department of Physics, 4056 Basel, Switzerland 


\title{
Dr. A. Devižis
}

FTMC, State Research Institute Center for Physical Sciences and Technology, Department of Molecular Compound Physics, Saulètekio Avenue 3, LT-10257 Vilnius, Lithuania

Keywords: electrolyte, light-emitting electrochemical cell, emission zone, electrical model, super yellow

\begin{abstract}
In light-emitting electrochemical cells (LECs), the position of the emission zone (EZ) is not predefined via a multilayer architecture design, but governed by a complex motion of electrical and ionic charges. As a result of the evolution of doped charge transport layers that enclose a dynamic intrinsic region until steady state is reached, the EZ is often dynamic during turn-on. For thick sandwich polymer LECs, a continuous change of the emission colour provides a direct visual indication of a moving EZ. Results from an optical and electrical analysis indicate that the intrinsic zone is narrow at early times, but starts to widen during operation, notably well before the electrical device optimum is reached. Results from numerical simulations demonstrate that the only precondition for this event to occur is that the mobilities of anions $\left(\mu_{\mathrm{a}}\right)$ and cations $\left(\mu_{\mathrm{c}}\right)$ are not equal, and the direction of the EZ shift dictates $\mu_{\mathrm{c}}>\mu_{\mathrm{a}}$. Quantitative ion profiles reveal that the displacement of ions stops when the intrinsic zone stabilizes, confirming the relation between ion movement and EZ shift. Finally, simulations indicate that the experimental current peak for constant-voltage operation is intrinsic and the subsequent decay does not result from degradation, as commonly stated.
\end{abstract}




\section{Introduction}

The simultaneous occurrence of electronic and mobile ionic charge in organic materials has numerous applications in technologies ranging from organic electrochemical transistors to batteries, actuators, sensors and displays. ${ }^{[1-3]}$ Arguably the best studied device application of the mixed electronic/ionic conduction in organic materials is the light-emitting electrochemical cell (LEC), and after 25 years of research the basic operation principles seem to have been firmly established. ${ }^{[4-10]}$

For a conjugated polymer LEC, a single solution-processed emissive polymer layer that contains an electrolyte is sandwiched between two electrodes. When a voltage is applied, the ions form electronic double layers (EDLs) at both electrodes that facilitate electronic charge injection. The reduced and oxidized polymer repeating units are electrostatically stabilized by opposite ionic charges, resulting in highly conductive $\mathrm{n}$ - and $\mathrm{p}$-doped regions at the cathode and anode, respectively, which grow inside the film. Over time, a p-i-n junction develops where electrons and holes are injected from the electrodes into the active layer and travel along the doped regions to the intrinsic (i) zone where they recombine radiatively. Next to polymers, ionic transition metal complexes (iTCMs) and organic salts have been used as active LEC materials. ${ }^{[7,11-13]}$ For these materials, the ionic and electronic transport occurs in the same material, but the device operation mechanism can be described by the same underlying processes. $^{[14,15]}$

The developing $\mathrm{p}-\mathrm{i}-\mathrm{n}$ junction and the position of the zone where light is emitted (emission zone, EZ) can conveniently be followed by optical probing and photoluminescence experiments on so-called planar LECs that have a wide horizontal gap between the two electrodes. ${ }^{[6,15-24]}$ The summary of numerous studies is that the EZ in many cases is offcentred. In addition, it has been observed that the EZ is dynamic and shifts across the device until steady state is reached. ${ }^{[15,21,25,26]}$ 
For sandwich-type LECs the active film thickness is below $1 \mu \mathrm{m}$ and the dynamic formation of the p-i-n structure cannot be directly visualized with optical microscopy. For these devices, information about the EZ position could be obtained from a comparison of experimental emission spectra with optical simulations. ${ }^{[27-33]}$ Just like for planar LECs, it was found that the EZ in sandwich LECs in many cases is off-centred and can move during operation.

As a complementary method, the p-i-n dynamics in sandwich LECs is often studied with impedance spectroscopy (IS). IS measurements yield simultaneous information about the dynamics of electronic and ionic charges, covering a wide frequency range from typically $\mathrm{Hz}$ to several MHz. Furthermore, the change in capacitance level at intermediate frequencies is commonly related to the evolution of the intrinsic region with low conductivity. ${ }^{[11,34-40]}$ Studies on poly(para-phenylenevinylene)-type LECs combined IS measurement with equivalent circuit or drift-diffusion modelling, providing a solid understanding of the dynamic LEC junction. ${ }^{[34,36]}$ One conclusion is that the width of the low-conductive region must not be equal to the width of the region with high recombination, i.e. the EZ ${ }^{[36,38]}$ Despite these substantial achievements in the characterization and understanding of the dynamic $p$-i-n junction, it seems that a quantitative model that describes the often observed EZ shift in planar and sandwich LECs has not been presented so far. Off-centred EZs at equilibrium were explained by an asymmetry of electron and hole injection and transport. ${ }^{[1,42]}$ For planar LECs, the moving p-n junction was explained with further doping of the partially doped polymer after the junction has formed. ${ }^{[23]} \mathrm{EZ}$ shifts were related to imbalances in carrier injection, conductivity, doping speed or a combination of these ${ }^{[15]} \mathrm{EZ}$ shifts for short operation times were ascribed to incomplete formation of the doped zones and hence unbalanced carrier injection that balances later on. ${ }^{[28,29]}$ 
Here, we present a comprehensive experimental optical and electrical analysis of a moving EZ in polymer sandwich LECs that continued for more than 20 hours and extended over more than $30 \%$ of the active layer thickness. For active layer thicknesses above $\sim 200$ $\mathrm{nm}$, the continuous change of the emission colour provided a direct visual indication of the moving EZ and the time-dependent EZ position was determined from angular emission measurements, combined with optical simulation. The transient capacitance increased first, passed a maximum and decreased to a lower steady-state value. This indicated that the intrinsic zone initially got smaller but increased again during device operation. Interestingly, the intrinsic zone width started to increase again well before the electrical device optimum was reached, i.e. before the voltage minimum in a constant-current experiment, or before the current maximum when using a constant-voltage driving mode. As commonly observed for constant-voltage driven LECs also the current, and consequently the luminance, decreased after passing a maximum.

We explained our observations of a moving EZ and a transient capacitance peak with results from numerical electrical drift-diffusion simulation and found that the sole precondition for these events to occur is that the mobilities of the anion and cation $\left(\mu_{\mathrm{a}}, \mu_{\mathrm{c}}\right)$ are not equal. Time-of-flight secondary ion mass spectrometry (ToF-SIMS) ion profiles at different times during operation showed that the displacement of anions stopped when the capacitance leveled off, confirming the relation between ion movement and the observed EZ shift. As an important insight into the background science, simulation showed that the experimental current trend observed for constant-voltage driven devices is intrinsic to the LEC operation and does not result from (unspecified) device degradation, as commonly assumed. Device degradation mechanisms prevail only after the ionic movement has stopped. 


\section{Results}

We investigated LECs with a phenyl-substituted poly(para-phenylenevinylene) copolymer termed super yellow (SY) as emitting material, intermixed with an electrolyte consisting of the salt lithium trifluoromethanesulfonate $\left(\mathrm{Li}^{+} \mathrm{CF}_{3} \mathrm{SO}_{3}{ }^{-}\right)$and a hydroxyl-capped oligoether, trimethylolpropane ethoxylate (TMPE). ${ }^{[43]}$ The chemical structures and the simple device architecture (glass/indium tin oxide (ITO)/active layer/Al or Ag top electrode) are shown in Figure S1 of the Supporting Information. In all following spatially resolved graphs and images, ITO is on the left and the metal electrode on the right side.

Previously, we optimized the efficiency $\left(11.6 \mathrm{~cd} \mathrm{~A}^{-1}\right)$ of this device in terms of materials composition ( $\left.\mathrm{SY}: \mathrm{TMPE}: \mathrm{Li}^{+} \mathrm{CF}_{3} \mathrm{SO}_{3}{ }^{-}=1: 0.075: 0.0225\right)$ and active layer thickness $(70 \mathrm{~nm}) .{ }^{[4]}$ Interestingly, a complex dynamic change in colour became apparent when studying devices with much thicker active layers, Figure 1a. Pictures were taken with an optical microscope while the $430 \mathrm{~nm}$ thick device was biased with a constant current of +7.7 $\mathrm{mA} \mathrm{cm}{ }^{-2}$, where the positive bias was applied to the ITO. The same trend is observed in Figure $1 \mathrm{~b}$ as a valley in the luminance, and the minimum luminance value was at the same point in time when the colour shifted from red to green, where it appeared also the darkest. The effect seems to be more pronounced when the active layer thickness was increased. On the other hand, the voltages in Figure 1c did not change drastically over time, the only trend observable is a slight increase of the minimal voltage, which scaled roughly linear with thickness (Figure S2a, Supporting Information).

As observed before for that particular materials system, ${ }^{[44]}$ it must be noted that the sample-to-sample variation was rather large, despite an apparent constant experimental device fabrication procedure. Data for the $430 \mathrm{~nm}$ thick device in Figure 1 show that the valley of the luminance was reached after $5 \mathrm{~h}$ and the corresponding voltage was $7.5 \mathrm{~V}$. From a larger set of LECs (Figure S3, Supporting Information), we found that the average time 
when the luminance valley was reached was $(7.5 \pm 2.9) \mathrm{h}$, and the corresponding average voltage was $(8.5 \pm 1) \mathrm{V}$. Therefore, we unravel in the following the basic mechanisms responsible for the observed dynamic change in colour and identify trends rather than claim exact numbers. This will be especially important when comparing experimental data with results from optical and electrical simulations.

Data in Figure 1 suggest an optical phenomenon as the reason for the observed change in colour. As explained in previous work, LECs are heavily influenced by the microcavity effect. ${ }^{[31,32,44]}$ Here, we expanded the optical simulations of reference [44] to an active layer thickness of $1000 \mathrm{~nm}$ as shown in Figure 1d and 1e, where Figure 1d shows the perceived colour and Figure 1e the luminance observed perpendicular to the emitting surface. The position of a Gaussian profile emitter with a width at half maximum of $20 \mathrm{~nm}$ was swept from ITO at emitter position 0 to the metal electrode at a relative emitter position of 1 . The resulting pattern appears as an interference effect due to the feedback of the emitted electromagnetic field of the dipole-like emitter that is reflected at the metal electrode. ${ }^{[45-47]}$ When the same data are plotted as an absolute distance from the metal electrode (Figure S2b and S2c, Supporting Information), it is evident that the effect depends only on the distance of the emitter to the metal electrode. The luminance valleys (Figure 1e) overlap with the red/green colour bands (Figure 1d) because interference is suppressing parts of the spectra, such that it is perceived as another colour. The complete optical model is described in the Supporting Information.

By comparing the colour change over time in Figure 1a with the colour bands in Figure 1d, an immediate hypothesis is that the region where excitons recombine under emission of light shifted with biasing time over a large distance of more than $100 \mathrm{~nm}$. Furthermore, the sequence of the change in colour is yellow-red-green-yellow, which dictates the movement to proceed from the metal electrode towards ITO. To quantify the EZ shift, we 
measured angular emission spectra from $-85^{\circ}$ to $85^{\circ}$ in $5^{\circ}$ steps while biasing a $430 \mathrm{~nm}$ and a $240 \mathrm{~nm}$ thick device with a constant current of $7.7 \mathrm{~mA} \mathrm{~cm}^{-2}$. Data were fitted with the optical model (see above) ${ }^{[31,44]}$ and the resulting emitter position over time is shown in Figure S2d, Supporting Information. These data were included as arrows into Figure 1d and 1e for clarity. Similar undulating luminance transients for SY LECs (using a different ion conductor and different materials composition) have recently been reported, but the optical analysis was restricted to steady state. ${ }^{[32,33]}$

For the $240 \mathrm{~nm}$ thick device after an operation time of $7 \mathrm{~h}$, the EZ shifted very close to the centre of the active film, whereas for the $430 \mathrm{~nm}$ thick device and after an operation time of $15 \mathrm{~h}$, the EZ was still slightly off-centred at a relative position of $\sim 0.6$ (Figure $1 \mathrm{~d}, 1 \mathrm{e}$ ). From Figure 1a, however, it can be seen that the change in colour for longer operation times continued and the emitted colour after $25 \mathrm{~h}$ was red. When extrapolating the arrow in Figure $1 \mathrm{~d}$ to the red colour band, it can be seen that the EZ for the $430 \mathrm{~nm}$ thick device after long operation time and close to steady state (see below) arrived also at a relative emitter position of $\sim 0.5$. From this we conclude that in our LECs the steady-state EZ was in the centre, largely independent of the active layer thickness. A centred EZ implies similar electronic mobility values $\left(\mu_{\mathrm{e}} \approx \mu_{\mathrm{h}}\right)$, or more precise a balance in the combination between hole and electron mobilites in the doped and intrinsic regions as well as charge injection, as will be confirmed from numerical simulations results below.

To investigate the emitter position and EZ shift, a number of experiments were carried out with $430 \mathrm{~nm}$ thick LECs. First, operated devices were short-circuited for at least $72 \mathrm{~h}$, i.e. the metal contact and ITO were connected with a metal wire. After that, the device was operated for a second time and the original sequence of the change in colour was observed again (Figure S4, Supporting Information). This means that during the time of non-use the pand n-regions de-doped and complete ion relaxation occurred. When the device was short- 
circuited for only $24 \mathrm{~h}$, the change in colour happened as well but started already from the colour red (emitter position $\sim 0.75$, Figure 1d), indicating a partial ion relaxation.

Reverse driving, i.e. when the negative bias was applied at the ITO, resulted in fast device degradation when using an $\mathrm{Al}$ electrode due to electrochemical reactions at the SY/Al interface. ${ }^{[48]}$ However, with an Ag electrode reverse biasing was possible. The surprising finding was that when the device turned on the emitted colour was green and the sequence of the change in colour was green-red-yellow, i.e. the opposite of the forward biased device (Figure S4, Supporting Information). We did not quantify from which position the emission started in that case, but from Figure 1d it gets clear that the EZ was now shifting from the ITO towards the Ag electrode. These observations demonstrate the dynamic LEC behaviour and show that the change in colour is not due to, for example, a trivial device degradation process. 
a)

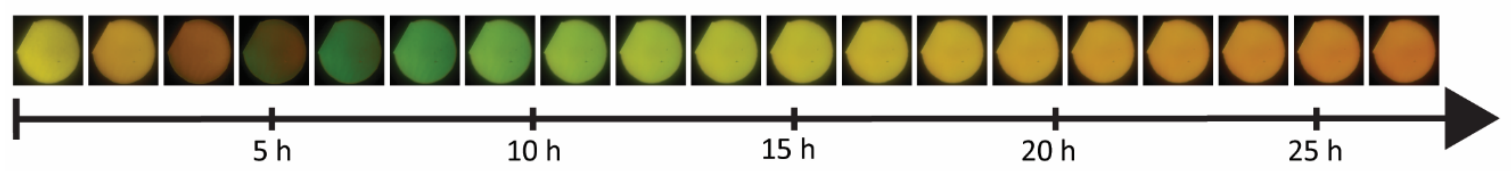

b)

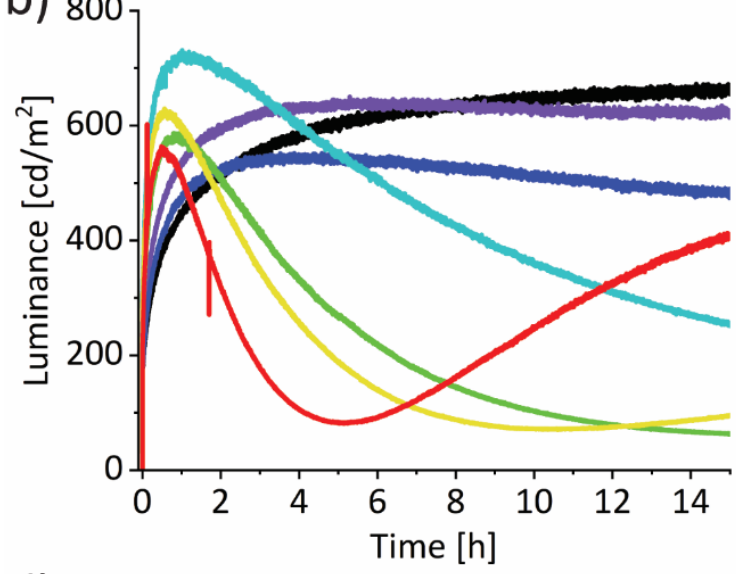

d)

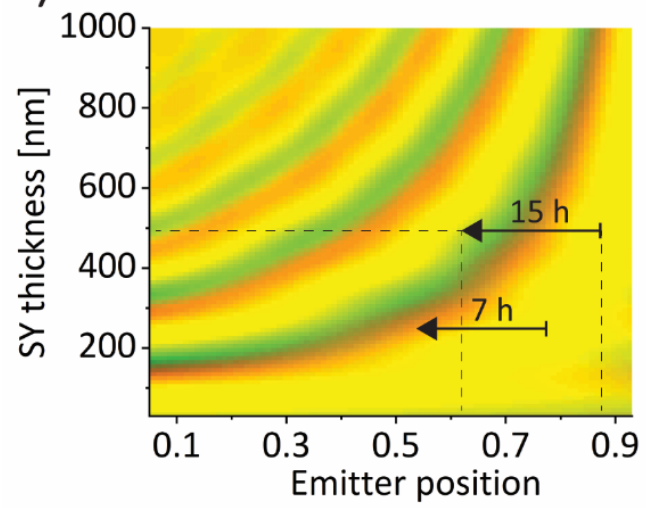

c)

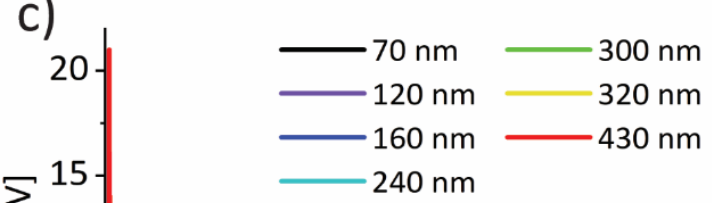

e)

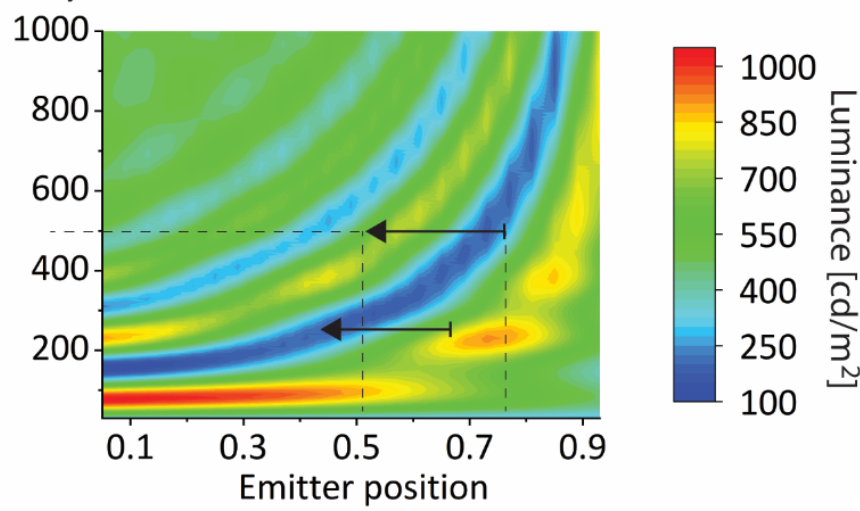

Figure 1. A change in colour over time is observed in (a) for a constant-current driven LEC with an active layer thickness of $430 \mathrm{~nm}$. (b) Luminance transients and (c) voltage transients for LECs with different active layer thicknesses. Optical simulations of the perceived colour (d) and luminance (e) at a $0^{\circ}$ observer angle demonstrate the dependence on active layer thickness and emitter position. Experimental emitter position shifts from angular emission measurements are indicated by arrows in (d) and (e).

Figure 2a shows the capacitance vs. frequency dynamics, which was calculated from the imaginary part of the admittance from IS measurements taken from the pristine device 
and during constant-current operation. In the pristine, undoped device, the whole active layer is intrinsic and the extracted capacitance $\left(4.8 \mathrm{nF} \mathrm{cm}{ }^{-2}\right)$ at $10^{7} \mathrm{~Hz}$ represents the geometrical capacitance. A slight increase of the capacitance to $7.1 \mathrm{nF} \mathrm{cm}{ }^{-2}$ at $10 \mathrm{~Hz}$ occurred, which can be attributed to charges that penetrate further into the device. ${ }^{[36]}$ During operation and after 1 $\mathrm{h}$ at lower frequencies, the capacitance has increased to a maximum value. This increase is related to the formation of the doped zones, resulting in a smaller intrinsic region, or more precisely to a shrinkage of the low conductivity part of the device. ${ }^{[36]}$ The threshold frequency at roughly $10^{3} \mathrm{~Hz}$ is the frequency where charges start to have enough time to reach the interface between the doped and intrinsic region, thus contributing to the capacitance. For longer measurement times up to $14 \mathrm{~h}$ the capacitance at low frequencies continuously decreased, indicating that the width of the intrinsic region increased again.

The simultaneously measured voltage, capacitance and light intensity transients are plotted in Figure $2 \mathrm{~b}$. The spikes in the voltage and photodiode response measurements are due to the $40 \mathrm{~s}$ breaks when the IS measurement was carried out at $0 \mathrm{~V}$, and can be attributed to a slight device relaxation during that period. The origin of these spikes is discussed in the Supporting Information.

The light intensity was measured with a photodiode. Because the temporal emission spectra are not precisely known, the exact conversion into a radiance (respectively luminance) transient is not possible. However, we detail in the Supporting Information (Figure S5) that the trends are the same and that the difference between peak and valley values varies by $\sim 15 \%$ at most. The light intensity peaked at around $2 \mathrm{~h}$, before the minimum of the voltage was reached at around $5 \mathrm{~h}$. Such behaviour is usually explained by increased exciton quenching when the narrowing intrinsic region gets in close contact with the doped zones. ${ }^{[11]}$ In our case, however, the observed light intensity decrease is due to interference effects while the EZ is shifting (Figure 1). 
Surprisingly, and in contrast to the current understanding of the functioning of LECs, also the capacitance peaked (after $1 \mathrm{~h}$ ) much before the minimum of the voltage was attained (Figure 2b). Generally, the transient voltage minimum is related to a device situation where the doped zones have fully developed, the intrinsic region is at its narrowest width and, consequently, the capacitance should be at the maximum. This observation was independent of the metal electrode (Al vs. Ag), of the active layer thickness, and also recurred for operated devices after relaxation (Figure S6, Supporting Information).

As the evolution of our LECs took place over a period of many hours, we supposed that the dynamics is dictated by the slow movement of the ions through the device. We performed ToF-SIMS experiments on $430 \mathrm{~nm}$ thick devices to determine the $\mathrm{CF}_{3} \mathrm{SO}_{3}{ }^{-}$ distribution throughout the film as function of the operation time. Recently, we operated LECs inside a ToF-SIMS spectrometer and cooled the sample to liquid nitrogen temperature before quantitative depth profiling was performed. ${ }^{[48]}$ For $60 \mathrm{~nm}$ thick devices, we found that the initial anion distribution was inhomogeneous with a peak at the ITO.$^{[48]}$ The same result was measured here for devices that contained an Al or Ag top electrode and the anions accumulated during spin coating and film formation preferentially at the bottom electrode (Figure 2 and Figure S7, Supporting Information). For this situation and when a positive bias is applied to the ITO, the anions would already be "at the right electrode" and their distribution would not change much during operation. Therefore, we studied the anion distribution for negative-biased (-7.7 $\mathrm{mA} \mathrm{cm}^{-2}$ at ITO) LECs with an Ag electrode; as explained above, such devises are stable when operated under a reverse bias.

Already after $10 \mathrm{~min}$, the $\mathrm{CF}_{3} \mathrm{SO}_{3}{ }^{-}$content at the ITO side has decreased and anions have started to pile up in a very narrow distribution at the Ag electrode. This can be interpreted as the start of the EDL formation. This ion displacement process continued for longer operation times and after around $10 \mathrm{~h}$, the ITO side was completely depleted and the 
anions transferred to the Ag side. In the bulk of the film, the anion concentration remained low throughout, except of a small anion peak in the thickness range of $\sim 400 \mathrm{~nm}$ to $420 \mathrm{~nm}$ that grew over an operation time of $20 \mathrm{~h}$.

We measured the voltage and capacitance trends for an ITO/SY(430 nm)/Ag LEC for reverse-biased operation conditions (Figure S7, Supporting Information), and observed similar transients as shown in Figure $2 \mathrm{~b}$ for a forward-biased LEC containing an Al electrode. The capacitance increased in the beginning, passed through a maximum (after $2.2 \mathrm{~h}$ ) and decreased afterwards. Also, the capacitance peaked before the minimum of the voltage was reached (after $3.3 \mathrm{~h}$ ). Interestingly, after around $10 \mathrm{~h}$ the capacitance levelled off and stayed almost constant during prolonged operation. From ToF-SIMS results, it can be seen that at this time also the final anion distribution has largely developed. This is a strong indication that the EZ shift indeed is dictated by the ionic displacement and that the overall device dynamics ends when the ionic distribution is in equilibrium. 


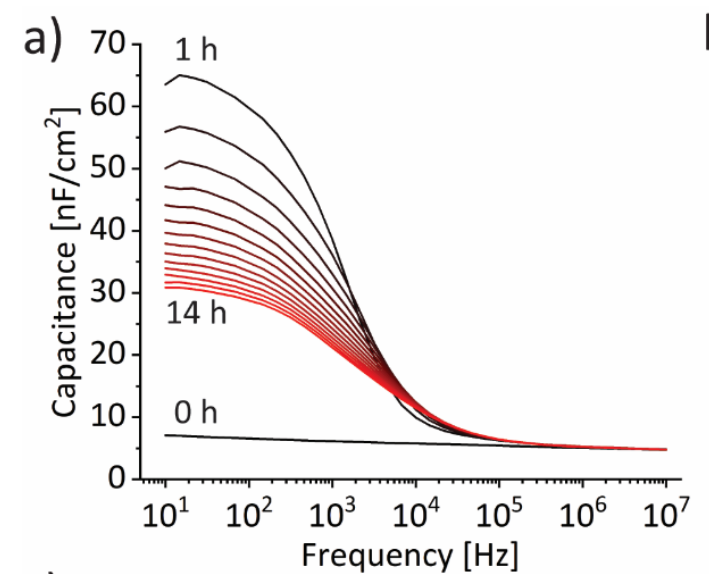

c)

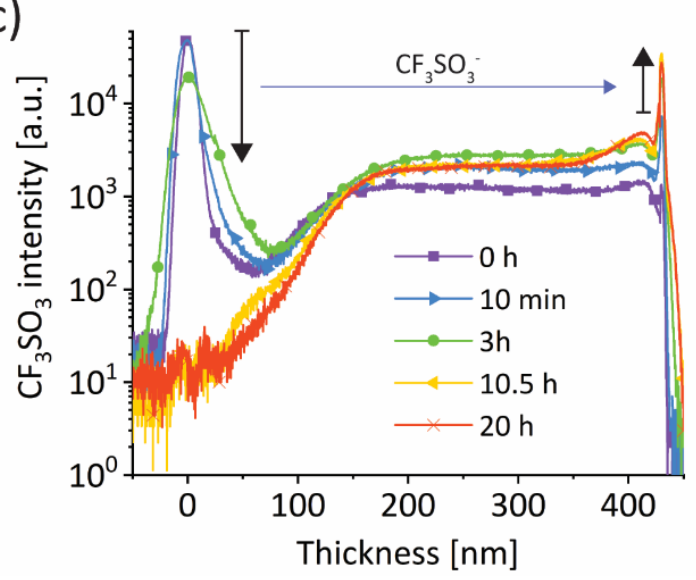

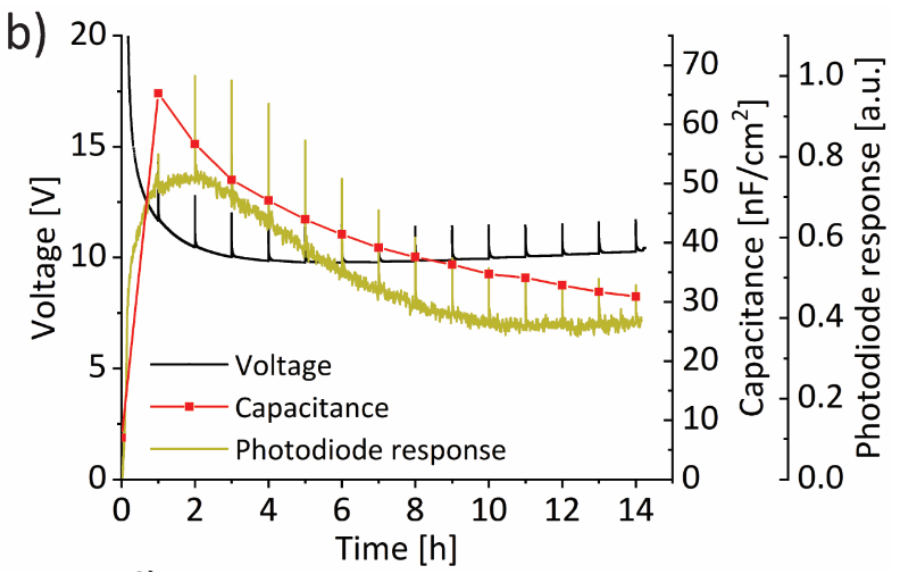

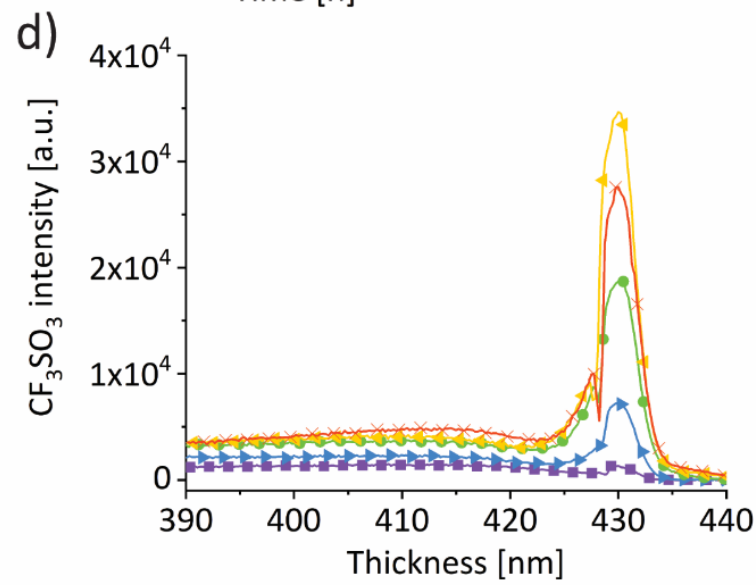

Figure 2. Capacitance, voltage, light intensity and ToF-SIMS measurements on $430 \mathrm{~nm}$ thick devices. The capacitance vs. frequency sweeps in (a) were measured every hour on a device with an $\mathrm{Al}$ electrode, while a constant current of $+7.7 \mathrm{~mA} \mathrm{~cm}^{-2}$ was applied at the ITO. The points at $10 \mathrm{~Hz}$ are plotted as transient in (b), together with the measured voltage and light intensity. ToF-SIMS measurements $(\mathrm{c}, \mathrm{d})$ were performed on a device with an Ag electrode and a reverse bias of $-7.7 \mathrm{~mA} \mathrm{~cm}^{-2}$ applied at the ITO.

Data presented so far were measured on constant-current driven devices. We performed measurements on constant-voltage driven LECs to confirm that the essential device dynamics is independent of the operation mode. Results from capacitance vs. frequency sweeps and from the current and light intensity trends of a $430 \mathrm{~nm}$ thick LEC operated at a constant voltage of $8 \mathrm{~V}$ are shown in Figure $3 \mathrm{a}$ and $3 \mathrm{~b}$. In Figure $3 \mathrm{a}$, the 
threshold frequency during the first few hours changed from $\sim 10^{3} \mathrm{~Hz}$ to $\sim 2 \times 10^{4} \mathrm{~Hz}$ because of a conductivity increase of the doped zones. As observed for constant-current driven devices (Figure 2), the capacitance passed through a maximum before the electrical device optimum, i.e. the maximum of the current, was reached. A similar observation was reported for a sandwich polymer LEC using a related materials system. ${ }^{[36]}$ Also the light intensity in Figure $3 \mathrm{~b}$ showed the same variation in time and the change in colour could clearly be observed (data not shown). However, a straightforward interpretation of the change in colour for constant-voltage driven devices is more involved because the change of the light intensity due to the EZ shift is superimposed on the variation of the light intensity due to the continuously changing current. In Figure $3 \mathrm{~b}$, the light intensity decrease between $\sim 10 \mathrm{~h}$ and $25 \mathrm{~h}$ had a much smaller decreasing slope than the current, which is probably a sign of an increase of the light outcoupling efficiency due to the shift of the EZ during that period. After $25 \mathrm{~h}$, the decrease of the light intensity followed exactly the current trend, which suggests that the EZ shift has stopped. Results from a further constant-voltage driven device where a clear luminance valley appeared are shown in Figure S8, Supporting Information. 
a)
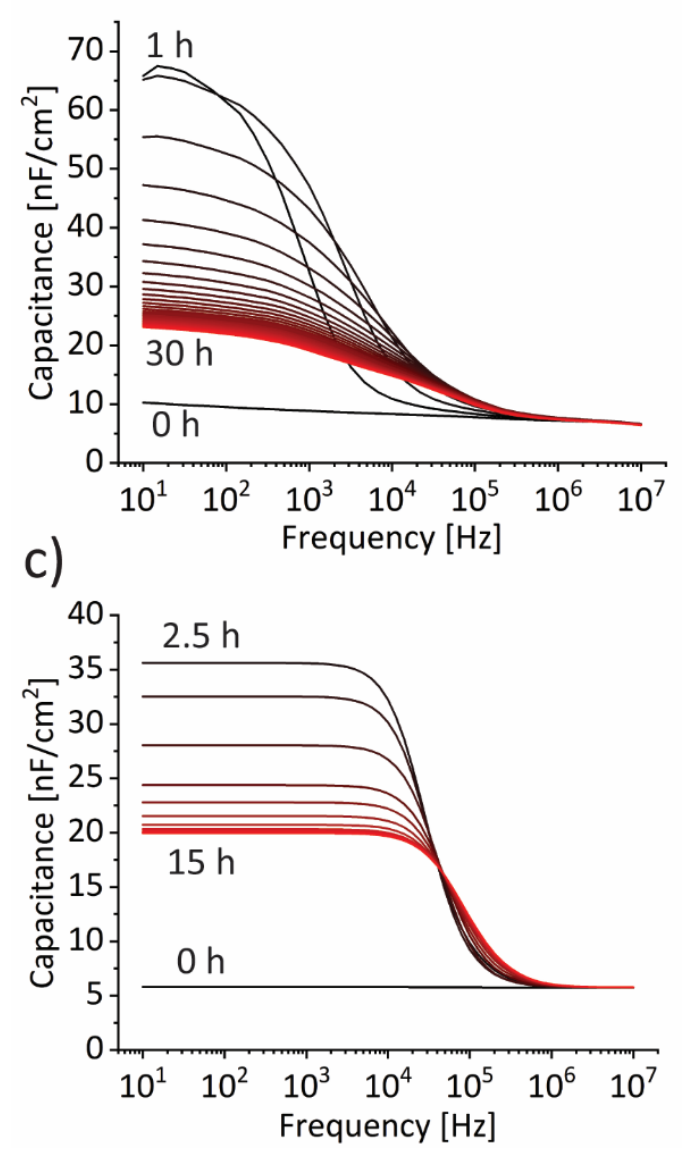

b)
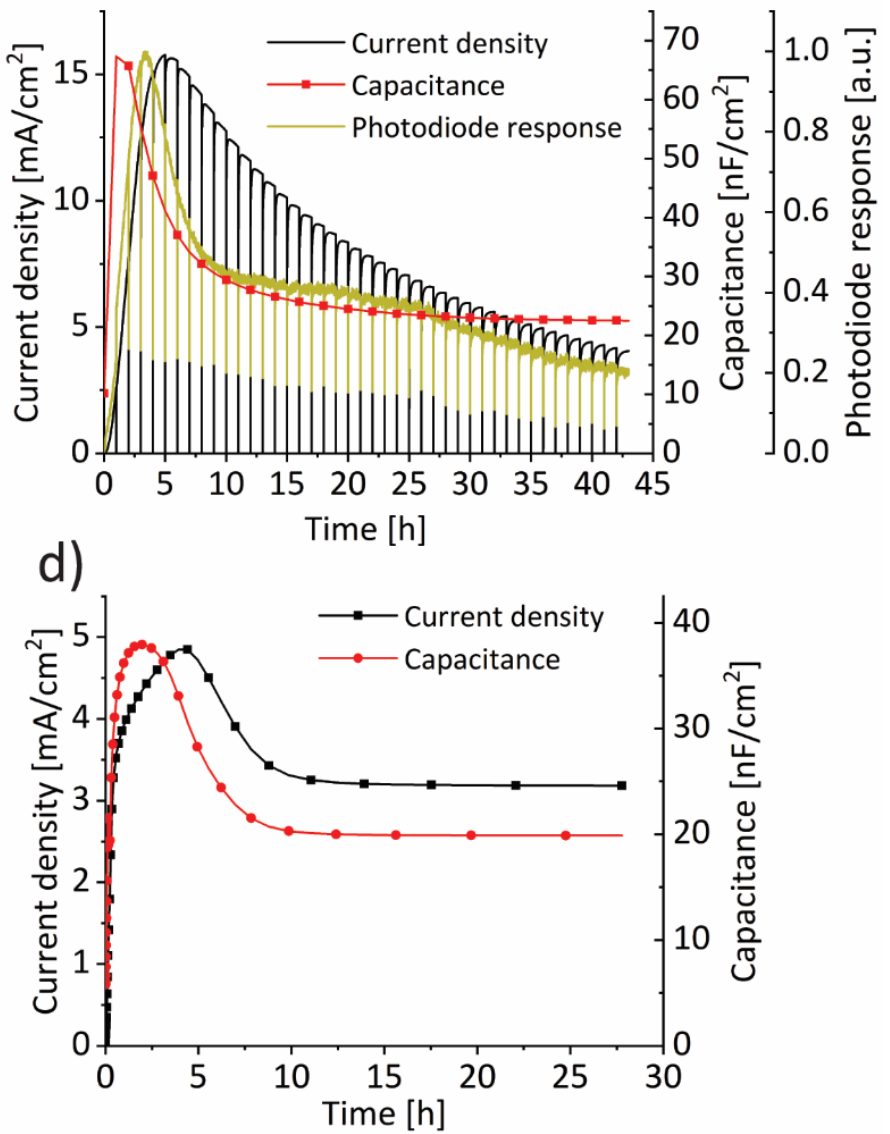

Figure 3. (a) Capacitance vs. frequency sweeps measured every hour and (b) transients for the capacitance at $10 \mathrm{~Hz}$, current and light intensity for a $430 \mathrm{~nm}$ thick LEC driven at a constant $8 \mathrm{~V}$ bias. Results from drift-diffusion simulations on this device are shown in (c) and (d). For the simulation, $\mu_{\mathrm{e}}=\mu_{\mathrm{h}}$, equal injection barriers for electrons and holes, and $\mu_{\mathrm{a}}=\mu_{\mathrm{c}} / 10$ was chosen, other parameters are compiled in Table S1, Supporting Information.

\section{Discussion}

Due to the complex and simultaneous motion of ions and electronic charges during operation, an intuitive explanation for the observed EZ shift in LECs is difficult. Therefore, we resorted to an analysis of the device dynamics by numerical drift-diffusion modelling performed by 
applying a constant voltage. Results of the transient simulation are shown in Figure $3 \mathrm{c}$ and $3 \mathrm{~d}$. The good agreement with the measurement is apparent and the simulation could reproduce the essential experimental features. To obtain that level of agreement between experiment and modelling, the only but necessary simulation condition was to choose a different mobility parameter for the two ionic species, and the direction of the EZ shifts required a higher value for the cation mobility, $\mu_{\mathrm{c}}>\mu_{\mathrm{a}}$. Note that no device degradation effects were included in the model, meaning the experimentally observed current and associated light intensity decrease is intrinsic. The simulated current decay from the peak value is indeed substantial and on the order of $35 \%$. This is a surprising result because a current decay in sandwich LECs for longer operation times so far has been mainly attributed to effects of - often unspecified - device degradation. The phenomenon seems not to be restricted to sandwich LECs, and similar current transients have been presented for planar LECs before. ${ }^{[6,17]}$ Of course, this does not imply that no device degradation is occurring. For example, the experimental current in Figure $3 b$ is continuously decreasing while the simulated current levels off after around $10 \mathrm{~h}$ of operation. As described above, we believe that after an experimental operation time of $\sim 25 \mathrm{~h}$ the EZ shift has ended, and we ascribe the further current and light intensity decrease to degradation.

Figure 4 shows the simulated device situation at different points of operation. The charge recombination profile (Figure 4a) is equivalent to the EZ and the current density is the area of the recombination profile, multiplied by the elementary charge q. For short operation times, the profile is narrow and situated close to the metal electrode. Over time, its width expands and shifts towards the ITO.

The simulated charge distribution profiles are shown in Figures $4 b-f$. Figure $4 \mathrm{~b}$ shows the experimental ToF-SIMS ion profile before operation, and very little electronic charge is present inside the device. Already after $0.3 \mathrm{~h}$ (Figure 4c), the EDLs at both electrodes are 
established and a small hole and electron current starts to develop. After 2.75 h (Figure 4d), the fast moving cations have completely displaced towards the metal electrode, while a fraction of the slow anions is still present in the bulk of the film. The charge recombination profile, which is proportional to the product of hole and electron density, is very narrow. This device situation corresponds to the time where the capacitance is highest. For longer operation time (Figures $4 \mathrm{e}$ and $4 \mathrm{f}$ ), the anions slowly drift towards the bottom electrode and the recombination zone gets wider. The recombination current peaks after an operation time of $5.5 \mathrm{~h}$. The intrinsic thickness transient calculated from the simulated capacitance by the plate capacitor formula $C=\varepsilon_{0} \varepsilon_{r} A / d$ correlates well with the recombination profile and the width defined by a certain electron and hole charge density, as explained in Figure S9, Supporting Information. The current peaks, however, when the area under the recombination profile curve is largest. The simulation confirms that the capacitance maximum must not coincide with the current maximum.

Details of the simulations and the parameter selection are described in the Supporting Information. In brief, the model solves the coupled continuity equations for the electronic and ionic charges and Poisson's equation as a function of time until steady state has been reached. For several time steps, the capacitance at $0 \mathrm{~V}$ is simulated by calculating the current response to a sinusoidal voltage. We summarize here the most important conclusions: (i) The initial ion density profile has no fundamental influence on the simulation outcome. In the simulations presented in Figure 4 the initial ion distribution was chosen based on ToF-SIMS measurements. For a homogeneous initial ion distribution, the current dynamics is slower but the overall EZ shift direction is the same. Experimentally, this statement is confirmed from data shown in Figure S4 and S10, Supporting Information. (ii) When $\mu_{\mathrm{c}}>\mu_{\mathrm{a}}$, the EZ shift direction is from the metal electrode towards ITO, for $\mu_{\mathrm{a}}>\mu_{\mathrm{c}}$ the shift direction is reversed. (iii) For $\mu_{\mathrm{c}}=\mu_{\mathrm{a}}$ and our experimental initial ion profile the EZ shift occurs from the ITO 
towards the metal electrode. For $\mu_{\mathrm{c}}=\mu_{\mathrm{a}}$ and a constant ion profile, no EZ shift occurs. Statements (ii) and (iii) are valid for any value of $\mu_{\mathrm{e}}$ and $\mu_{\mathrm{h}}$. (iv) Imbalanced $\mu_{\mathrm{e}}$ and $\mu_{\mathrm{h}}$ or charge injection has an influence on the current level and the position of the EZ in steady state is off-centred, but the EZ shift direction is solely determined by $\mu_{\mathrm{c}}$ and $\mu_{\mathrm{a}}$. More precise, our experimentally found centred $\mathrm{EZ}$ is the result of balanced charge mobility in combination with injection and, for example, an imbalance of $\mu_{\mathrm{h}}$ and $\mu_{\mathrm{e}}$ can be compensated by a reciprocal imbalance in charge injection. (v) For a given average ion density, the steady state situation does not depend on the ion mobility values and the pristine ion profile. 

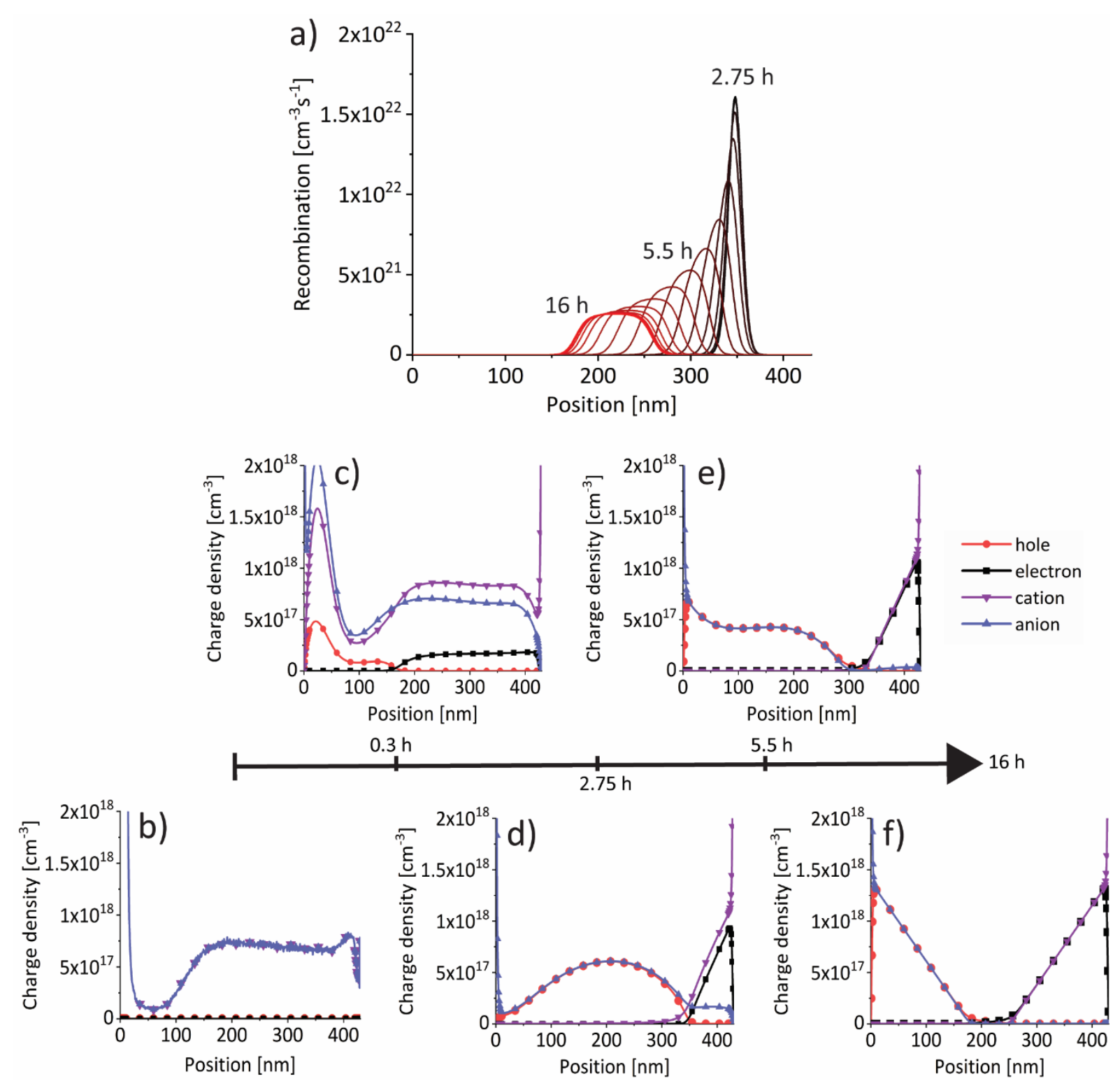

Figure 4. Simulation details for a $430 \mathrm{~nm}$ thick LEC. (a) The charge recombination profile corresponds to the position of the EZ. (b) - (f) Simulated charge distribution profiles for anions, cations, holes and electrons. For the simulation, $\mu_{\mathrm{e}}=\mu_{\mathrm{h}}$, equal injection barriers for electrons and holes, and $\mu_{\mathrm{a}}=\mu_{\mathrm{c}} / 10$ was chosen. An equal mobility value for electrons and holes and a balanced injection results in a centred EZ at steady state (f) - as observed experimentally - independent of the values for $\mu_{\mathrm{a}}$ and $\mu_{\mathrm{c}}$. 
Finally, we performed measurements and simulations for LECs with a $70 \mathrm{~nm}$ thick active layer thickness. Data in Figure 1d show that in such a case the emitted colour will always be yellow, independent of the EZ. Therefore, a shifting EZ does not result in a change of the emission colour. Figure 5a and 5b show the capacitance, current and light intensity trends for a constant $+4 \mathrm{~V}$ driven LEC. Compared to the $430 \mathrm{~nm}$ thick device (Figure 3a), the capacitance in Figure 5a decreased at high frequencies because of a higher (geometrical) capacitance and an increase of the RC time constant. As observed for the $430 \mathrm{~nm}$ thick devices, the capacitance peaked before the current and levelled off for long operation times. These features were nicely reproduced by the simulation. In contrast to thick LECs, the light intensity maximum was now after the current peak. ${ }^{[44,49]}$ We suggest this is a signature of the moving EZ that occurs also in thin-film devices. Figure 1e shows that for thicknesses below around $100 \mathrm{~nm}$ the luminance increases strongly when the EZ shifts from the metal electrode towards ITO. Therefore, for the thin device the maximum light intensity was measured after the current peak. Comparison between experiment and simulation suggests that also for thinfilm LECs the measured current decrease before about $8 \mathrm{~h}(\sim 10 \%)$ is intrinsic and only the succeeding decrease is due to device degradation. 
a)

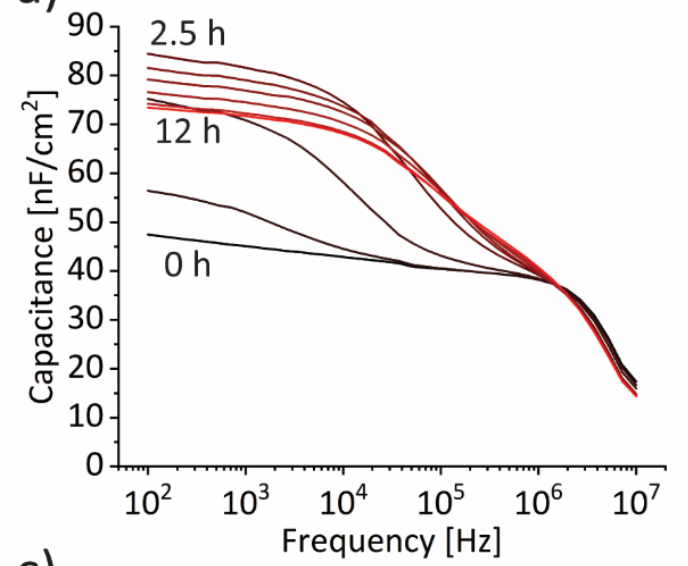

c)

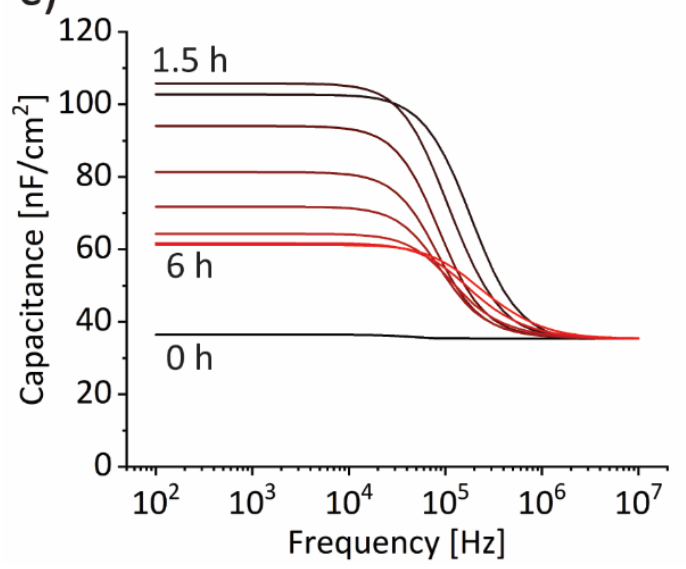

b)

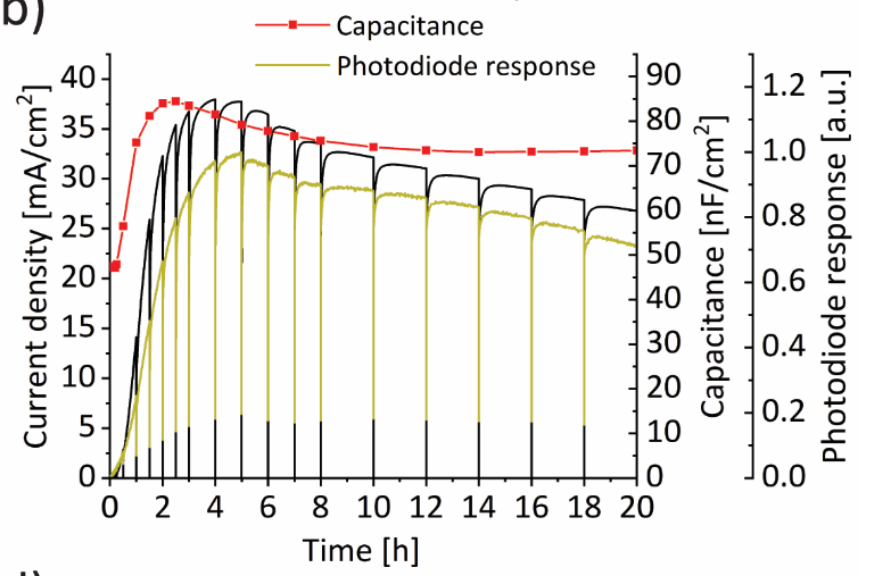

d)

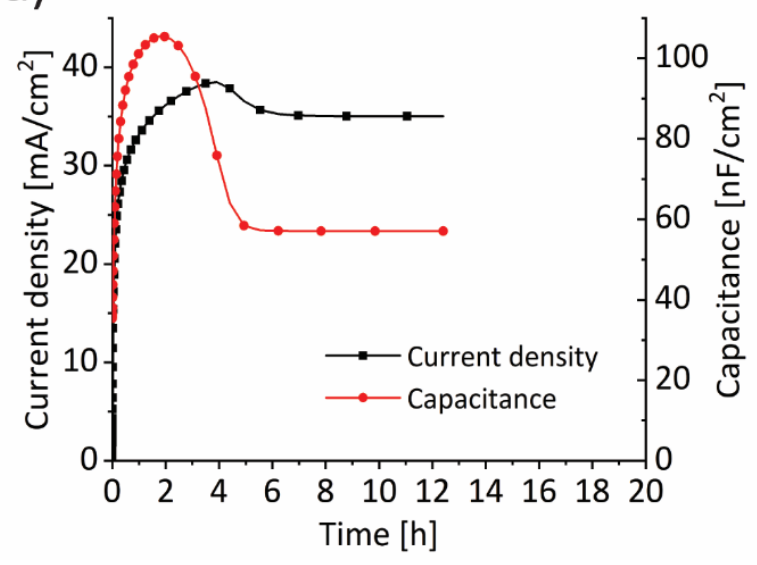

Figure 5. (a) Capacitance vs. frequency sweeps and (b) transients for the capacitance at 10 $\mathrm{Hz}$, current and light intensity for a $70 \mathrm{~nm}$ thick LEC driven at a constant $+4 \mathrm{~V}$ bias. Results from drift-diffusion simulations are shown in (c) and (d). For the simulation, $\mu_{\mathrm{e}}=\mu_{\mathrm{h}}$, equal injection barriers for electrons and holes, and $\mu_{\mathrm{a}}=\mu_{\mathrm{c}} / 10$ was chosen, other parameters are compiled in Table S1, Supporting Information.

Within the approximations of the model, we could thus demonstrate that a different mobility value for anions and cations is a sufficient requirement to explain the observed shift and widening of the EZ for our polymer LECs. However, simulations also showed that the transient and final EZ situation depends on the exact values chosen for the electronic mobilities and the charge injection. This means that a non-constant mobility during operation 
can also result in a continuous change of the EZ. Indeed, our assumption of constant $\mu_{\mathrm{e}}$ and $\mu_{\mathrm{h}}$ is in the general case not correct because mobilities may depend on the temperature, on the electric field as well as the doping level and thus possibly change continuously during operation. ${ }^{[36,50-53]}$

Time dependent EZ shifts in iTMC sandwich LECs were explained with imbalanced carrier injection and transport at early times that balanced during operation. Importantly, the EZ stabilized quickly while the current continuously increased for a much longer time. ${ }^{[29]}$ This is different from our observations. We also observed very similar luminance and voltage trends for LECs with an Al or Ag metal electrode over a measurement period of $16 \mathrm{~h}$ (Figure S15, Supporting Information). This finding points to different critical factors that determine the EZ dynamics in our LECs, because effects of injection barriers and imbalanced charge injection should become apparent during early operation times when the EDLs form and the doped regions start to grow. Furthermore, from simulation results (Figure S12 and S14, Supporting Information), we find that while imbalanced electronic charge mobilities indeed result in an off-centred EZ, the influence on the calculated capacitance level is marginal.

For constant-current driven polymer LECs, Gao et al. reported that at elevated temperature, either due to internal or external heating, a p-n junction can relax into a p-i-n junction. A rapidly increasing voltage during operation indicated that the width of the resistive intrinsic zone increased, resulting in a strong increase of the luminance because in a wider intrinsic region less excitons are quenched by the nearby doped regions. ${ }^{[54-56]}$

In agreement with other reports, ${ }^{[57-59]}$ we indeed also measured a considerable device temperature increase during operation (Figure S16, Supporting Information). However, we mention observations that indicate that in our case the temperature increase was probably not the reason for the observed widening of the EZ over time. The capacitance trends did not 
correlate with the heat generated from the electric power, which is the product of voltage and current. The capacitance peaked before the voltage minimum (Figure 2b), meaning that the intrinsic region width started to increase before the minimum of the generated heat was reached. Likewise, for constant-voltage operation (Figure 3b), the intrinsic region width continued to grow when the heat load decreased after the current peak. Furthermore, when applying a constant current, the capacitance decrease and thus the increase of the intrinsic region levelled off while the slope of the voltage drift was increasing; this is especially evident from Figure S6a, Supporting Information.

\section{Conclusions}

We used a combination of experiments and simulation to demonstrate that the observed EZ shift and the measured capacitance trend in polymer sandwich LECs is due to an imbalance between the cation and anion mobility. The experimental determination of the initial, inhomogeneous ion profile was an essential ingredient of this work. In most simulation studies reported so far, a homogeneous initial ion distribution and $\mu_{\mathrm{c}}=\mu_{\mathrm{a}}$ were chosen as modelling parameters. For these conditions, no simulated EZ shift occurs over time. Our findings indicate that for constant-voltage operation the current decay from the peak is intrinsic and not (exclusively) due to device degradation, which has implication for further studies. For example, a differentiation between an intrinsic current decay and current decline due to device degradation can be made by operating a device twice, with a relaxation delay in between.

Our observations are not consistent with the hypothesis that the observed EZ shift over many hours is due to continuously changing electronic charge mobilities and injection barriers. However, we do not claim generality of our findings and other materials systems 
might behave differently. For example, iTMC LECs present a case where the large cation is essentially immobile. ${ }^{[15,40,61]}$ This has the consequence that the EDL formation at the cathode is slow and imbalanced charge injection can potentially prevail very long.

For lighting applications a fast turn-on and stable light source is desired.

Experimentally, this can be achieved when $\mu_{\mathrm{c}}=\mu_{\mathrm{a}}$, since an imbalance in the ion mobility values is the main cause for a shifting EZ and thus changing luminance with time. Furthermore, the EZ should be positioned in a region with high light outcoupling efficiency. The EZ position can be tuned by adjusting the electronic mobility values or the charge injection ratio, respectively - the ionic mobility values have no influence on the EZ position in steady state.

\section{Experimental Section}

Dried $\left(24 \mathrm{~h}, 0.1 \mathrm{mbar}, 40{ }^{\circ} \mathrm{C}\right) \mathrm{SY}$ (Merck) and dried $\left(24 \mathrm{~h}, 0.1 \mathrm{mbar}, 160{ }^{\circ} \mathrm{C}\right) \mathrm{Li}^{+} \mathrm{CF}_{3} \mathrm{SO}_{3}{ }^{-}$ (Sigma-Aldrich) together with dried (24 h, 0.1 mbar, room temperature) TMPE (SigmaAldrich, average $M_{n} 450$ ) were separately dissolved in anhydrous tetrahydrofuran (THF, Sigma Aldrich). The concentrations for salt and TMPE were $10 \mathrm{mg} \mathrm{mL}^{-1}, \mathrm{SY}$ is described below. Precursor solutions were stirred for $7 \mathrm{~h}$ at $60{ }^{\circ} \mathrm{C}$ inside a glove box $\left(\mathrm{H}_{2} \mathrm{O}<1 \mathrm{ppm}, \mathrm{O}_{2}\right.$ $<20 \mathrm{ppm})$. The precursor solutions were mixed in mass ratios of 1:0.1:0.03

(SY:TMPE: $\mathrm{Li}^{+} \mathrm{CF}_{3} \mathrm{SO}_{3}{ }^{-}$) and were then stirred for at least $17 \mathrm{~h}$ at $60{ }^{\circ} \mathrm{C}$. Before spin coating, solutions were let to cool down for $20 \mathrm{~min}$. The spin coating recipes for the film thicknesses $70,110,160,240,300,320$, and $430 \mathrm{~nm}$ were $[(5,2000),(6.5,2000),(8,2000),(10,2000)$, $(12,2000),(10,1000),(12,1000)]$, in $\left[\left(\mathrm{mg} \mathrm{SY} \mathrm{mL}^{-1} \mathrm{THF}, \mathrm{rpm}\right)\right]$, the acceleration (in $\left.\mathrm{rpm} / \mathrm{s}\right)$ was equal to the speed in all cases, and the runtime was $60 \mathrm{~s}$ in all cases. Non-filtered solutions were used for film coating. Layer thicknesses were measured with an Ambios XP1 
profilometer. The indicated film thicknesses above $(70 \mathrm{~nm}-430 \mathrm{~nm})$ are average values from several measurements, but we note that for thicker films (above $\sim 200 \mathrm{~nm}$ ) the thickness was not always constant over the sample film and we found thickness variations of around $\pm 10 \%$ when measuring at different positions. Aluminum or silver top electrodes $(70 \mathrm{~nm})$ were thermally evaporated through a shadow mask defining 8 cells with an active area of 3.1 or 7.1 $\mathrm{mm}^{2}$ per substrate.

For luminance measurements, devices were placed in an airtight holder and were measured under nitrogen atmosphere outside the glove box at room temperature using a factory calibrated Konica Minolta LS-110 luminance meter with a close-up lens 110. The reflection of the top cover glass of the holder was not considered. The refractive indices for the intrinsic SY layer were taken from reference [60] and were confirmed by simulation of experimental transmission spectra, measured with a Varian Cary $50 \mathrm{UV}-$ vis spectrometer. ${ }^{[4]}$ The photoluminescence spectrum was measured by fluorescence spectroscopy (Horiba Jobin Yvon Fluorolog).

The angular dependent EL measurement was performed with a prototype of the Phelos measurement system (Fluxim AG, Switzerland). Optical and electrical simulations were performed with Setfos 5.0 (Fluxim AG, Switzerland). Simulation procedures and parameters are described in the Supporting Information. Impedance measurements at $0 \mathrm{~V}$ with an alternating $70 \mathrm{mV}$ signal to determine the capacitance transients were performed on the Paios measurement system (Fluxim AG, Switzerland), as well as the corresponding current and light intensity transients. The light intensity was measured with a photodiode as photovoltage. The relation between the measured photovoltage and the corresponding radiance/luminance is explained in the Supporting Information.

ToF-SIMS measurements were performed on a ToF-SIMS.5 instrument from IONTOF, Germany, operated in the spectral mode using a $25 \mathrm{keV} \mathrm{Bi}_{3}{ }^{+}$primary ion beam with an ion 
current of $0.7 \mathrm{pA}$. For the ToF-SIMS measurements, LECs were operated in-situ and cooled down to liquid nitrogen temperature after a defined time in order to conduct dual beam depth profiling. For depth profiling, a $1 \mathrm{keV} \mathrm{Cs}^{+}$sputter beam with a current of $70 \mathrm{nA}$ was used. The raster area of the sputter beam was $500 \mu \mathrm{m} \times 500 \mu \mathrm{m}$, and the mass-spectrometry was performed on an area of $100 \mu \mathrm{m} \times 100 \mu \mathrm{m}$ in the centre of the sputter crater. During ToF-SIMS dual beam depth profiling a low-energy electron flood gun was used for charge compensation. The ToF-SIMS method was established in reference [48].

\section{Supporting Information}

Supporting Information is available from the Wiley Online Library or from the author.

\section{Acknowledgements}

M. D. and A. S. contributed equally to this work. Financial support from the Swiss

Commission for Technology and Innovation (project perolec 18468.1 PFNM-NM) and the Swiss National Science Foundation (grant CR23I2-162828) is acknowledged.

\section{Conflict of Interest}

The authors declare no conflict of interest.

Received: ((will be filled in by the editorial staff))

Revised: ((will be filled in by the editorial staff)) Published online: ((will be filled in by the editorial staff)) 


\section{References}

[1] Iontronics, Ionic Carriers in Organic Electronic Materials and Devices, Eds. J. Leger, M. Berggren, S. Carter, CRC Press, 2011.

[2] E. W. H. Jager, O. Inganäs, I. Lundström, Science 2000, 288, 2335.

[3] J. Rivnay, S. Inal, A. Salleo, R. M. Owens, M. Berggren, G. G. Malliaras, Nature Rev. Mater. 2018, 3, 17086.

[4] Q. Pei, G. Yu, C. Zhang, Y. Yang, A. J. Heeger, Science 1995, 269, 1086.

[5] S. Tang, L. Edman, Top. Curr. Chem. (Z) 2016, 374, 40.

[6] S. van Reenen, P. Matyba, A. Dzwilewski, R. A. J. Janssen, L. Edman, M. Kemerink, J. Am. Chem. Soc. 2010, 132, 13776.

[7] R. D. Costa, E. Ortí, H. J. Bolink, F. Monti, G. Accorsi, N. Armaroli, Angew. Chem. Int. Ed. 2012, 51, 8178.

[8] S. B. Meier, D. Tordera, A. Pertegás, C. Roldán-Carmona, E. Ortí, H. J. Bolink, Mater. Today 2014, 17, 217.

[9] E. Fresta, R. D. Costa, J. Mater. Chem. C 2017, 5, 5643.

[10] J. Gao, ChemPlusChem 2017, 82, 1.

[11] S. Jenatsch, L. Wang, M. Bulloni, A. C. Véron, B. Ruhstaller, S. Altazin, F. Nüesch, R. Hany, ACS Appl. Mater. Interfaces 2016, 8, 6554.

[12] A. Pertegás, D. Tordera, J. J. Serrano-Pérez, E. Ortí, H. J. Bolink, J. Am. Chem. Soc. 2013, 135, 18008 .

[13] S. Jenatsch, L. Wang, N. Leclaire, E. Hack, R. Steim, S. B. Anantharaman, J. Heier, B. Ruhstaller, L. Penninck, F. Nüesch, R. Hany, Org. Electronics 2017, 48, 77.

[14] S. van Reenen, T. Akatsuka, D. Tordera, M. Kemerink, H. J. Bolink, J. Am. Chem. Soc. 2013, 135, 886. 
[15] S. B. Meier, S. van Reenen, B. Lefevre, D. Hartmann, H. J. Bolink, A. Winnacker, W. Sarfert, M. Kemerink, Adv. Funct. Mater. 2013, 23, 3531.

[16] J.-H. Shin, N. D. Robinson, S. Xiao, L. Edman, Adv. Funct. Mater. 2007, 17, 1807.

[17] S. van Reenen, P. Matyba, A. Dzwilewski, R. A. J. Janssen, L. Edman, M. Kemerink, Adv. Funct. Mater. 2011, 21, 1795.

[18] Y. Hu, J. Gao, J. Am. Chem. Soc. 2011, 133, 2227.

[19] F. AlTal, J. Gao, Phys. Status Solidi RRL 2015, 9, 77.

[20] P. Matyba, K. Maturova, M. Kemerink, N. D. Robinson, L. Edman, Nature Mater. 2009, 8,672 .

[21] Y. Hu, J. Gao, Appl. Phys. Lett. 2006, 89, 253514.

[22] J. Fang, P. Matyba, N. D. Robinson, L. Edman, J. Am. Chem. Soc. 2008, 130, 4562.

[23] N. D. Robinson, J. Fang, P. Matyba, L. Edman, Phys. Rev. B 2008, 78, 245202.

[24] D. Hohertz, J. Gao, Adv. Mater. 2008, 20, 3298.

[25] Q. Pei, Y. Yang, G. Yu, C. Zhang, A. J. Heeger, J. Am. Chem. Soc. 1996, 118, 3922.

[26] T. Wågberg, P. R. Hania, N. D. Robinson, J.-H. Shin, P. Matyba, L. Edman, Adv. Mater. 2008, 20, 1744.

[27] H.-C. Su, ChemPlusChem 2018, 83, 197.

[28] R. Sun, C.-T. Liao, H.-C. Su, Org. Electronics 2014, 15, 2885.

[29] T.-W. Wang, H.-C. Su, Org. Electronics 2013, 14, 2269.

[30] Y.-P. Jhang, H.-F. Chen, H.-B. Wu, Y.-S. Yeh, H.-C. Su, K.-T. Wong, Org. Electronics 2013, 14, 2424.

[31] S. Jenatsch, M. Regnat, R. Hany, M. Diethelm, F. Nüesch, B. Ruhstaller, ACS Photonics 2018, 5, 1591.

[32] E. M. Lindh, P. Lundberg, T. Lanz, J. Mindemark, L. Edman, Sci. Rep. 2018, 8, 6970.

[33] E. M. Lindh, P. Lundberg, T. Lanz, L. Edman, Sci. Rep. 2019, 9, 10433. 
[34] A. Munar, A. Sandström, S. Tang, L. Edman, Adv. Funct. Mater. 2012, 22, 1511.

[35] Y. A. Davis, P. P. Crooker, N. M. Haegel, Y. Yoshioka, J. D. MacKenzie, Appl. Phys. Lett. 2011, 99, 233306.

[36] S. van Reenen, R. A. J. Janssen, M. Kemerink, Adv. Funct. Mater. 2012, 22, 4547.

[37] I. H. Campbell, D. L. Smith, C. J. Neef, J. P. Ferraris, Appl. Phys. Lett. 1998, 72, 2565.

[38] A. Devižis, S. Jenatsch, M. Diethelm, V. Gulbinas, F. Nüesch, R. Hany, ACS Photonics 2018, 5, 3124.

[39] S. B. Meier, D. Hartmann, A. Winnacker, W. Sarfert, J. Appl. Phys. 2014, 116, 104504

[40] L. D. Bastatas, M. D. Moore, J. D. Slinker, ChemPlusChem 2018, 83, 266.

[41] Y. Yang, Q. Pei, Appl. Phys. Lett. 1996, 68, 2708.

[42] J. A. Manzanares, H. Reiss, A. J. Heeger, J. Phys. Chem. B 1998, 102, 4327.

[43] S. Tang, L. Edman, J. Phys. Chem. Lett. 2010, 1, 2727.

[44] M. Diethelm, Q. Grossmann, A. Schiller, E. Knapp, S. Jenatsch, M. Kawecki, F. Nüesch, R. Hany, Adv. Optical Mater. 2019, 7, 1801278.

[45] H. Becker, S. E. Burns, R. H. Friend, Phys. Rev. B 1997, 56, 1893.

[46] R. H. Friend, R. W. Gymer, A. B. Holmes, J. H. Burroughes, R. N. Marks, C. Taliani, D. D. C. Bradley, D. A. Dos Santos, J. L. Brédas, M. Löglund, W. R. Salaneck, Nature 1999, 397, 121

[47] S. Höfle, T. Lutz, A. Egel, F. Nickel, S. W. Kettlitz, G. Gomard, U. Lemmer, A. Colsmann, ACS Photonics 2014, 1, 968.

[48] M. Kawecki, R. Hany, M. Diethelm, S. Jenatsch, Q. Grossmann, L. Bernard, H. J. Hug, ACS Appl. Mater. Interfaces 2018, 10, 39100. 
[49] K. Strassel, S. P. Ramanandan, S. Abdolhosseinzadeh, M. Diethelm, F. Nüesch, R. Hany, ACS Appl. Mater. Interfaces 2019, 11, 23428.

[50] V. I. Arkhipov, E. V. Emelianova, P. Heremans, H. Bässler, Phys. Rev. B 2005, 72, 235202.

[51] Y. Murat, G. Wantz, S. Fasquel, J.-Y. Laurent, T. Maindron, L. Hirsch, Proc. of SPIE 2016, 9941, 994129.

[52] S. R. Tseng, Y. S. Chen, H. F. Meng, H. C. Lai, C. H. Yeh, S. F. Horng, H. H. Liao, C. S. Hsu, Syn. Metals 2009, 159, 137.

[53] S. van Reenen, R. A. J. Janssen, M. Kemerink, Org. Electronics 2011, 12, 1746.

[54] Y. Zhang, Y. Hu, J. Gao, Appl. Phys. Lett. 2006, 88, 163507.

[55] Y. Zhang, J. Gao, J. Appl. Phys. 2006, 100, 084501.

[56] S. Hu, J. Gao, Adv. Mater. Techn. 2018, 3, 1800229.

[57] L. Edman, M. Pauchard, B. Liu, G. Bazan, D. Moses, A. J. Heeger, Appl. Phys. Lett. 2003, 82, 3961.

[58] L. Edman, Electrochimica Acta 2005, 50, 3878.

[59] G. Yu, Y. Cao, M. Andersson, J. Gao, A. J. Heeger, Adv. Mater. 1998, 10, 385.

[60] T. Lanz, E. M. Lindh, L. Edman, J. Mater. Chem. C 2017, 5, 4706.

[61] J. Mindemark, L. Edman, J. Mater. Chem. C 2016, 4, 420. 


\section{Table of contents entry}

In polymer sandwich light-emitting electrochemical cells, a difference between the mobility of anions and cations results in a shift of the light emission zone that continues for more than 20 hours and extends over more than $30 \%$ of the active layer thickness. Contrary to popular belief, the charge recombination zone is narrow at early times and widens during ongoing operation.

Keyword Light-emitting electrochemical cell

Matthias Diethelm, Andreas Schiller, Maciej Kawecki, Andrius Devižis, Balthasar Blülle, Sandra Jenatsch, Evelyne Knapp, Quirin Grossmann, Beat Ruhstaller, Frank Nüesch, and Roland Hany

\section{The Dynamic Emission Zone in Sandwich Polymer Light-Emitting Electrochemical}

\section{Cells}

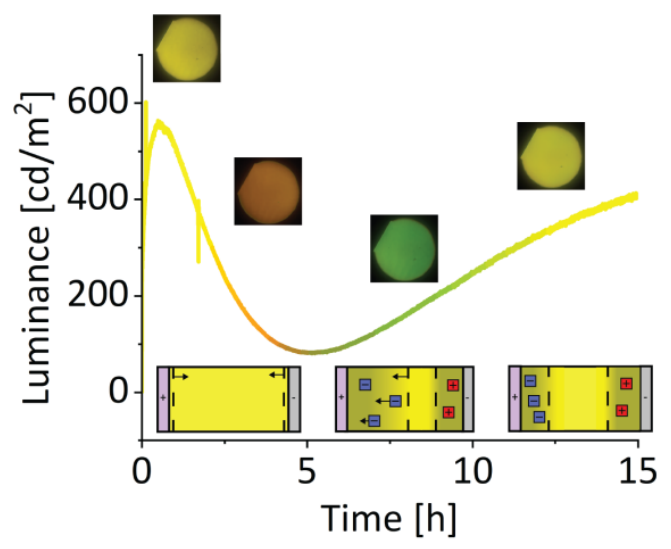

